\title{
De l'influence des Mémorables (I 4, IV 3) sur le De Natura deorum (II) de Cicéron
}

\section{Louis-André Dorion}

\section{(2) OpenEdition}

1 Journals

\section{Édition électronique}

URL : https://journals.openedition.org/philosant/659

DOI : 10.4000/philosant.659

ISSN : 2648-2789

Éditeur

Éditions Vrin

\section{Édition imprimée}

Date de publication : 1 novembre 2016

Pagination : 181-208

ISBN : 978-2-7574-1472-9

ISSN : 1634-4561

\section{Référence électronique}

Louis-André Dorion, "De l'influence des Mémorables (I 4, IV 3) sur le De Natura deorum (II) de Cicéron », Philosophie antique [En ligne], 16 | 2016, mis en ligne le 01 novembre 2018, consulté le 03 décembre 2022. URL : http://journals.openedition.org/philosant/659; DOI : https://doi.org/10.4000/philosant. 659

\section{(c) (†) $\odot$}

Creative Commons - Attribution - Pas d'Utilisation Commerciale - Pas de Modification 4.0 International - CC BY-NC-ND 4.0

https://creativecommons.org/licenses/by-nc-nd/4.0/ 


\section{DE L'INFLUENCE DES MÉMORABLES (I 4, IV 3) SUR LE DE NATURA DEORUM (II) DE CICÉRON}

Louis-André DORION

Université de Montréal

RÉSUMÉ : Les chapitres I 4 et IV 3 des Mémorables, où Socrate expose une téléologie anthropocentrique et une conception élaborée de la providence divine qui gouverne l'univers, ont exercé une profonde influence sur les stoïciens. Les Anciens ont reconnu cette influence, mais les Modernes ont tardé à la reconnaître, tantôt parce qu'ils ont considéré que ces chapitres des Mémorables étaient en réalité des interpolations d'origine stoïcienne, tantôt parce qu'ils ont attribué à Diogène d'Apollonie la téléologie exposée par Socrate. Fort heureusement, les commentateurs récents ne cherchent plus à déposséder Xénophon de la paternité de ces deux chapitres et l'on peut donc tenter de déterminer quelle fut leur postérité chez les stoïciens. L'étude qui suit s'applique à mettre en lumière les nombreux recoupements que l'on observe entre le texte des Mémorables (I 4 et IV 3) et le livre II du De natura deorum de Cicéron, où Balbus expose longuement la théologie stoïcienne. Il appert que l'influence des Mémorables sur ce texte de Cicéron est beaucoup plus considérable qu'on ne l'a reconnu jusqu'à maintenant.

SUMMARY : Chapters I.4 and IV.3 of the Memorabilia, where Socrates presents an anthropocentric teleology and an elaborate conception of the divine providence that rules the universe, had a profound influence on the Stoics. The ancients were well aware of this influence, but modern acknowledgement of it was slow in coming, sometimes because these chapters of the Memorabilia were seen as interpolations of Stoic origin, sometimes because the teleology presented by Socrates was attributed to Diogenes of Apollonia. Fortunately, recent commentators no longer seek to deny Xenophon's authorship of these two chapters, and it is therefore possible to attempt to determine the extent of their legacy among the Stoics. The aim of the following study is to shed light on the numerous overlaps that can be observed between the text of the Memorabilia (chapters I.4 and IV.3) and book II of Cicero's De natura deorum, where Balbus offers a detailed presentation of Stoic theology. It appears that the influence of the Memorabilia on Cicero's text is much more important than has been recognized before now. 



\section{Rendre enfin à Xénophon ce qui lui appartient : le cas de Mem. I 4 et IV $3{ }^{*}$}

Nous avons conservé deux importants témoignages qui font état de l'influence que les Mémorables auraient exercée sur le fondateur du stoïcisme, Zénon de Citium. L'un de ces témoignages se trouve au début du livre VII de Diogène Laërce, qui est entièrement consacré au stoöcisme.

Il [scil. Zénon] devint donc élève de Cratès dans les circonstances suivantes. Alors qu'il importait de la pourpre de Phénicie, il fit naufrage près du Pirée. Étant monté à Athènes, déjà âgé de trente ans, il s'assit chez un libraire. Comme celui-ci faisait lecture du deuxième livre des Mémorables de Xénophon ( il demanda où vivaient de tels hommes. Cratès se trouva à passer juste au bon moment. Le libraire le lui désigna et dit : « C'est lui qu'il te faut suivre ». De ce jour, il devint l'auditeur de Cratès, manifestant de façon générale une grande ardeur à l'égard de la philosophie, bien qu'il éprouvât de la honte devant l'impudeur cynique'.

Il serait sans doute naiff de croire à l'historicité de cette anecdote dans la mesure où elle est probablement conçue pour établir un lien de filiation entre Socrate et les stoïciens, par l'intermédiaire des cyniques. Il n'empêche que la plupart des commentateurs ${ }^{2}$ la prennent très au sérieux et y voient un signe, parmi d'autres, de l'influence déterminante que les Mémorables auraient exercée sur Zénon. L'anecdote rapportée par Diogène Laërce rejoint un autre témoignage, celui de Sextus Empiricus. Après avoir longuement résumé, en le paraphrasant, le texte de Mem. I 4, 2-8, Sextus affirme que Zénon s'inspira de Xénophon pour avancer un argument sur l'existence de Dieu et la rationalité du monde ${ }^{3}$.

* J'ai grandement profité, pour la version finale de cette étude, des commentaires et des suggestions que m'a transmis Jean-Baptiste Gourinat. Je tiens à l'en remercier.

1. VII 2-3 (trad. Goulet 1999).

2. Cf. entre autres, Long 1988, p. 162-163.

3. «Zénon de Citium, trouvant son point de départ chez Xénophon (ảmò $\Xi \varepsilon v o \varphi \hat{\omega} v \tau o \varsigma$

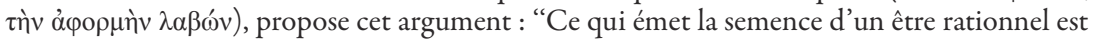


Nous avons donc deux témoignages qui font état de l'influence des Mémorables sur Zénon de Citium. Le fait qu'il s'agisse de Zénon, et non pas d'un stoïcien ultérieur, n'est pas à dédaigner, car nous aurions ainsi à faire à une influence « originelle » de Xénophon sur certaines positions-clés du stoïcisme naissant, et non pas à une influence tardive sur des points d'importance mineure. Je souligne au passage que ces deux témoignages ne parlent pas des Mémorables en général, mais chaque fois d'un livre en particulier. Il me paraît bien difficile, voire impossible, de déterminer ce que le livre II des Mémorables peut avoir transmis à Zénon, mais il est beaucoup plus facile de le déterminer dans le cas du livre I, puisqu'il s'agit à l'évidence des positions théologiques exposées en I 4.

Ces deux témoignages auraient dû encourager les interprètes à entreprendre des recherches dans le but d'identifier quelles sont les thèses développées dans les Mémorables qui auraient pu être reprises et réélaborées par les stoïciens. Or je ne connais aucun interprète qui se soit engagé dans cette voie au XIX ${ }^{\mathrm{e}}$ siècle et $S$ chleiermacher ${ }^{4}$ avait lui-même affirmé, dans une conférence prononcée en 1815, que le stoïcisme ne devait rien au Socrate de Xénophon. Soixante ans après Schleiermacher, A. Krohn s'est tout de même avisé qu'il y avait plusieurs recoupements entre les deux chapitres théologiques des Mémorables (I 4 et IV 3) et la téléologie stoïcienne. Mais plutôt que de reconnaître l'influence des Mémorables sur le stoïcisme, Krohn a soutenu que les chapitres théologiques des Mémorables étaient en réalité des interpolations d'origine stoïcienne, ce qui revient à dire, comme Schleiermacher mais autrement que lui, que Xénophon était décidément incapable d'exercer quelque influence que ce soit sur le stoïcisme ${ }^{5}$. Les thèses de Krohn exercèrent une réelle influence, puisque W. Gilbert, l'éditeur du texte des Mémorables dans la collection Teubner, s'est réclamé de Krohn pour justifier l'atéthèse pure et simple des chapitres I 4 et IV $3^{6}$. Trente ans après la publication de l'ouvrage de Krohn, K. Lincke a défendu les mêmes thèses dans un article influent ${ }^{7}$. Les

lui-même rationnel. Or le monde émet la semence d'un être rationnel. Donc le monde est rationnel. Ceci permet aussi de conclure que Dieu existe” » (Sextus Empiricus, Contre les Physiciens, I $101=$ M. IX 101). L'usage que Sextus Empiricus fait du texte de Xénophon a fait l'objet de deux études approfondies ( $c f$. Sedley 2005, p. 471-483 et Meijer 2007, p. 12-23).

4. Schleiermacher 1840, p. cxxxi-cxxxii.

5. Voir notamment Krohn 1875, les chapitres intitulés « Die Stoa in den Memorabilien » (p. 1-21) et « Die Kosmologie in den Memorabilien » (p. 46-60).

6. Dans la praefatio critica qui précède le texte des Mémorables, Gilbert écrit : «Id quoque mibi Krohn persuasit, non a Xenophonte scriptum hoc caput [scil. I 4] esse, maxime quod ea doctrina, ex qua anima humana pars videtur animae mundanae [...], Stoica est, non Socratica » (Gilbert 1891 p. xx). Voir aussi p. Ixii : « Nam cum alterum de providentia divina caput non Socraticum sit $(I, 4)$, suspicio oritur, hoc quoque caput $(I V, 3)$ non Socraticis, sed Stoicis assignandum esse, qui doctrinam de providentia ( $\pi$ oóvola) excoluerunt ; cf. Krohn. »

7. Lincke 1906. 
élucubrations de Lincke furent contestées dès 1907 par M. Barone ${ }^{8}$, mais ce court article n'encouragea pas pour autant les commentateurs à étudier les rapports entre Xénophon et le stoïcisme, sans doute parce que l'on demeurait réticent à voir en Xénophon le véritable auteur des chapitres théologiques des Mémorables. Mais plutôt que de voir dans ces deux chapitres des interpolations d'origine plus tardive, on fut enclin à les considérer comme les vestiges d'une doctrine téléologique développée par des philosophes antérieurs à Xénophon, notamment Diogène d'Apollonie.

Comme l'explique très bien $\mathrm{A}$. Laks dans son ouvrage sur Diogène d'Apollonie, « [1] a défense du texte incriminé [scil. Mem. I 4 et IV 3] devait dès lors rendre plausible l'existence d'une doctrine de la providence divine à haute époque, autrement dit, proposer un auteur à qui l'attribuer. Comme Socrate n'entrait pas en ligne de compte, parce qu'il n'est que le Socrate de Xénophon, et Xénophon pareillement, parce qu'il n'est que Xénophon, il fallait, pour authentifier les chapitres incriminés, chercher ailleurs. ${ }^{9} \gg$ Cette entreprise, qui ressortit à la Quellenforschung, est surtout illustrée par les travaux de F. Dümmler ${ }^{10}$ et de W. Theiler ${ }^{11}$, qui se sont efforcés d'identifier les sources de la conception de la providence divine exposée en Mem. I 4 et IV 3. Selon Dümmler et Theiler, la principale source de la doctrine téléologique que Xénophon prête à Socrate serait Diogène d'Apollonie. Cette interprétation s'est à ce point imposée que W. Jaeger, qui y souscrit entièrement ${ }^{12}$, n'hésite pas à parler du «Socrate-Diogène de Xénophon ${ }^{13}$ », comme si Socrate n'était rien de plus qu'un porte-parole de la doctrine téléologique de Diogène d'Apollonie $^{14}$. Dans un appendice à son édition commentée des fragments de Diogène, Laks a toutefois mis en lumière la fragilité et les faiblesses des hypothèses qui ont conduit à une telle conclusion ${ }^{15}$. La position de Laks fut

8. Barone 1907. Cf. aussi Festugière 1949, p. 78 n. 2 : « Je ne vois dans ces chapitres [scil. Mem. I 4 et IV 3] aucun trait qui dénote spécifiquement le stoïcisme : ni le Logos pénétrant toutes les parties de l'univers, ni la sympathie unissant entre eux tous les êtres du Kosmos, ni l'Heimarménè inflexible réglant tout le cours des affaires humaines. »

9. Laks 1983, p. 250.

10. Dümmler 1889, p. 96-165.

11. Theiler 1925 , p. 13-36.

12. Jaeger 1943, II, p. $31 ; 1966$, p. 179-183.

13. Jaeger 1966, p. 180.

14. Plusieurs commentateurs ont souscrit sans réserve aux conclusions de Theiler ( $c f$., entre autres, Festugière 1949, II, p. 77 n. 5, 78 n. 2). Plus près de nous, G. Vlastos (1991, p. 162 n. 26) ajoute encore foi à la position de Theiler, telle qu'elle est transmise par Jaeger. Deux ans après la publication de l'ouvrage de Theiler, mais sans faire référence à ce dernier, Diès (1927, p. 533-536) attribue à Diogène d'Apollonie certains arguments développés en Mem. I 4 et IV 3.

15. Laks 1983 , p. $250-257$. 
par la suite corroborée par une étude indépendante de M. McPherran ${ }^{16}$ et, plus récemment, par un article de D. Sedley ${ }^{17}$.

Laks rappelle fort à propos, en conclusion de son analyse critique des positions défendues par Dümmler et Theiler, que K. Joël, dès 1893, « réduisait la téléologie anthropocentrique des deux chapitres [scil. Mem. I 4 et IV 3] à une conception utilitariste de la divinité, qui répondait primairement à des intérêts personnels profonds et spécifiquement xénophontiens ${ }^{18} \gg$. Il ne serait donc indispensable, pour rendre compte de la téléologie anthropocentrique de Mem. I 4 et IV 3, ni de chercher en aval et de conclure hardiment à une interpolation d'origine stoïcienne, ni de chercher en amont et d'attribuer hâtivement à Diogène d'Apollonie la paternité de la doctrine téléologique exposée par le Socrate de Xénophon. Dans une étude qui rend justice à l'intérêt et à la cohérence des arguments développés en Mem. I 4 et IV 3, McPherran ${ }^{19}$ s'efforce de démontrer que la doctrine téléologique des Mémorables ne doit pas être attribuée à Xénophon, mais à Socrate lui-même, quand bien même les dialogues socratiques de Platon ne renferment aucune doctrine de ce genre. Sedley parvient à la même conclusion que McPherran : c'est à Socrate lui-même, et non pas à ses prédécesseurs, qu'il faut attribuer les thèses développées en $M e m$. I 4 et IV $3^{20}$. C'est une position à laquelle je suis réticent à souscrire, car si une telle doctrine remonte au Socrate historique, comment se fait-il que nous n'en percevions chez Platon aucune trace avant le Phédon et des dialogues aussi tardifs que le Timée, le Philèbe et les Lois ? Or mon propos n'est pas de déterminer si la doctrine théo-téléologique exposée dans les Mémorables doit être attribuée, ou non, au Socrate historique, mais plutôt de présenter un exemple précis de l'influence que cette doctrine a pu exercer sur le stoïcisme. Il me suffit donc, pour les besoins de cette étude, que l'on rende enfin à Xénophon ce qui lui appartient, à savoir la paternité des chapitres I 4 et IV 3 des Mémorables.

C'est ma conviction profonde que la réhabilitation philosophique des Mémorables est une condition indispensable à l'examen serein de l'influence que cette œuvre a exercée sur des philosophes ultérieurs; cet examen est

16. « Socrates did not much rely on Diogenes in formulating the teleological account of the cosmos we find in the Memorabilia (an account that far exceeds Diogenes' in its range, detail, moral import, and argumentative sophistication) 》(McPherran 1994, p. 260). Cf. aussi Charlton 1991, p. 88.

17. Sedley 2005, p. 461 : «Les idées qui y sont exposées [scil. Mem. I 4] ne dérivent en effet pas de Diogène d'Apollonie, dont le rôle dans l'histoire de la pensée téléologique a été largement surestimé » (voir aussi p. $462-464$ et Sedley 2008, p. 331). Sedley (2005, p. 466 n. 13) s'oppose également à l'hypothèse de F. Decleva Caizzi (1966, p. 100-101), suivant laquelle Antisthène serait la source de Mem. I 4.

18. Laks 1983 p. 256.

19. McPherran 1994.

20. Sedley 2005, p. $467 ; 2008$, p. 318. 
impossible si l'on n'a que mépris pour les Mémorables, ou que l'on refuse d'y voir quoi que ce soit d'original. Et c'est d'ailleurs pour cette raison que les interprètes ont négligé, jusqu'à tout récemment, l'étude approfondie des rapports entre Xénophon et le stoïcisme. Les choses sont cependant, et fort heureusement, en train de changer. J. DeFilippo et P. Mitsis ont mis en lumière la continuité entre la conception socratique des lois non écrites et la théorie stoïcienne de la loi naturelle ${ }^{21}$; M. Erler voit dans un passage des Mémorables (I 2, 49-55) la préfiguration ou l'amorce de la conception stö̈cienne de l'oikeiosis ${ }^{22}$; enfin, D. Sedley a dernièrement publié plusieurs études qui soulignent l'influence des chapitres théologiques des Mémorables sur la téléologie ultérieure et sur les preuves stoïciennes de l'existence de $\mathrm{Dieu}^{23}$. Les études de Sedley sont de toute première importance, mais elles n'ont pas pour autant épuisé le sujet. Sa perspective est en effet très circonscrite dans la mesure où il s'intéresse surtout, voire exclusivement, au témoignage de Sextus Empiricus sur Mem. I 4, 2-8. Ce témoignage est précieux pour autant qu'il permet de comprendre comment Zénon s'est approprié le passage de Xénophon et comment il a répondu à certaines objections qui lui furent adressées par le mégarique Alexinus.

\section{Xénophon et Cicéron}

\section{L'influence de Mem. I 4 et IV 3 sur le De natura deorum}

S'il est vrai, comme l'affirme Sedley, que les chapitres théologiques des Mémorables avaient 《un statut canonique dans l'école stoïcienne $»^{24}$, on devrait s'attendre à trouver plusieurs traces de cette influence dans les textes stoïciens $^{25}$. Or non seulement les études de Sedley ne fournissent aucune référence, hormis le texte de Sextus Empiricus et quelques passages du $D e$ natura deorum de Cicéron ${ }^{26}$, à des textes stoïciens qui attesteraient cette influence des Mémorables, mais l'apparat des testimonia, dans l'édition Budé

21. DeFilippo \& Mitsis 1994.

22. Erler 2002.

23. Sedley 2005, 2007 et 2008.

24. Sedley 2005, p. 478 et p. 473 ; voir aussi Powers 2009, p. 264 : « There is reason to think that the "theological" portions of the Memorabilia were popular texts among the Stoics. »

25. Voir aussi Long 2013, p. 2-3 : «It is now widely agreed that Stoic moral philosophy was undertaken as a development of Socratic ethics, and Socrates' significance for the Stoics may have extended beyond ethics, for Stoic cosmo-theology seems to have drawn on Socrates'statements in Xenophon (Mem. 1.4.5-18, 4.3.2-18) about the divine design of the world and its human inhabitants. 》

26. Sedley (2005, p. 473) affirme que « le statut canonique propre à ce texte [scil. Mem. I 4] est par ailleurs confirmé par le passage de Cicéron déjà mentionné [scil. De natura deorum $=N D$ II 18] $\gg$. L'étude que je propose vise à confirmer, par la mise en lumière des nombreux recoupements entre les Mémorables et le livre II du De natura deorum, le bienfondé de cette affirmation de Sedley. 
du texte des Mémorables ${ }^{27}$, ne fait mention que de sept textes stoïciens ${ }^{28}$ qui feraient écho aux chapitres I 4 et IV 3 des Mémorables. Or cet apparat des testimonia peut être considérablement enrichi. Pour me limiter à un seul exemple, une lecture attentive du livre II du De natura deorum de Cicéron révèle en effet que l'influence des Mémorables sur ce texte ne se limite pas aux quatre passages ${ }^{29}$ identifiés dans l'apparat des testimonia.

Que l'on me permette d'ouvrir une parenthèse : pourquoi avoir choisi le livre II du De natura deorum comme exemple de l'influence exercée par les Mémorables sur le stoïcisme ? Pour les deux raisons suivantes : premièrement, c'est l'exposé le plus long et le plus complet sur la théologie stoïcienne, de sorte que l'on peut s'attendre à y trouver des traces de l'influence des Mémorables; deuxièmement, nous savons que Cicéron est un lecteur de Xénophon ${ }^{30}$; en effet, non seulement il fait directement référence à l'auteur des Mémorables au livre II du De natura deorum ${ }^{31}$, mais il a également traduit l'Économique dans sa jeunesse ${ }^{32}$. La sympathie marquée de Cicéron pour Xénophon ${ }^{33}$ est de toute première importance pour mon étude, car elle m'évite de courir le risque, et de commettre l'erreur méthodologique, qui consisterait à identifier des réminiscences des Mémorables chez un auteur dont nous ne serions même pas sûrs qu'il les ait lus.

Venons-en sans plus tarder au tableau des recoupements entre les chapitres théologiques des Mémorables, Platon et le livre II du De natura deorum.

27. Bandini \& Dorion 2000 et 2011.

28. Cf. (1) Mem. I 4, 2-8 et Sextus Empiricus, Contre les physiciens, I 92-103 (= M. IX 92-103) ; (2) Mem. I 4, 5-6 et Cicéron, ND II 56-57 ; (3) Mem. I 4, 7 et Épictète, fr. 23 Schenkl (= Stobée, IV 53, 29) ; (4) Mem. I 4, 8 et Cicéron, ND II 18 et III 27 ; (5) Mem. I 4, 11 et Cicéron, ND II 56 ; (6) Mem. IV 3, 13 et Cicéron, ND I 31 ; (7) Mem. IV 3, 16-17 et Épictète, Ench. XXXI 5.

29. Cf. $N D$ I 31, II 18, II 56-57, III 27.

30. À titre indicatif, on relève vingt-huit occurrences du nom «Xénophon » dans le corpus de Cicéron (Brutus 292; De oratore II 58 ; III ,139; Orator 32; 62; De inventione I, 31, 51 ; I 31, 52 (quater) ; Cato maior 30; 46; $58 ; 79$; De divinatione I, 52 ; I 53 ; De finibus II, 28, 92 ; De legibus II, 56 ; ND I 31 ; II, 18 ; III, 27 ; De officiis I, 32, 118 ; II, 24, 87 ; Tusculanae II, 26, 62 ; V, 34, 99 ; Epistulae ad familiares V,12, 7 ; Epistulae ad Quintum fratrem I, $1,22 ; \mathrm{I}, 2,7)$.

31. Cf. I 31, II 18, III 27.

32. Cf. Off. II 24, 87. Cicéron cite un passage de la Cyropédie (I 6, 25) en Tusc. II 26, 62.

33. Voir, entre autres, ce que Cicéron dit de Xénophon dans l'Orator : « J'ai même rencontré un orateur pour vouloir ressembler à Xénophon, dont le style, plus doux que le miel, j'en suis d'accord, est celui qui convient le moins au tumulte du forum » (32); voir aussi 62 : « les Muses semblent avoir parlé par la bouche de Xénophon » (trad. Bornecque 1921). 
Tableau des recoupements ${ }^{34}$

\begin{tabular}{|c|c|c|c|}
\hline Thème & Xénophon & Platon & $\begin{array}{l}\text { Cicéron et textes } \\
\text { d'inspiration stoïcienne }\end{array}$ \\
\hline $\begin{array}{l}\text { 1. Preuve de } \\
\text { l'existence de Dieu } \\
\text { par l'intelligence } \\
\text { cosmique (1) }\end{array}$ & I $4,2-8$ & $\varnothing$ & $\begin{array}{l}\text { Sextus Empiricus, } \\
\text { Contre les physiciens, } \\
\text { I 92-103 }\end{array}$ \\
\hline $\begin{array}{l}\text { 2. Les choses ne sont } \\
\text { pas le produit du } \\
\text { hasard, mais de } \\
\text { l'intelligence (1) }\end{array}$ & I 4,$4 ; 6 ; 9$ & $\begin{array}{c}\text { Lois X } \\
888 \mathrm{e}-889 \mathrm{~d}\end{array}$ & $\begin{array}{l}\text { ND II } 15 ; \text { II } 87-88 \text {; II } \\
93 \text {; II } 97 \text {; II } 115 \text {; II } \\
128 ; \text { II } 153 . \\
\text { - Épictète, I 6, } 7 \text {; I } 6 \text {, } \\
11 \text {; Philon, Prov. I } 42\end{array}$ \\
\hline $\begin{array}{l}\text { 3. Le monde procède } \\
\text { d'une intelligence } \\
\text { (1) }\end{array}$ & I $4,5-6$ & $\begin{array}{c}\text { Timée } \\
47 \mathrm{e}-48 \mathrm{a}\end{array}$ & ND II 16; II 56-57 \\
\hline $\begin{array}{l}\text { 4. L'homme a été } \\
\text { conçu par les dieux }\end{array}$ & $\begin{array}{c}\text { I } 4,5 ; \text { IV } 3 \\
11-12\end{array}$ & Timée 44c & ND II 4 ; II 64 \\
\hline $\begin{array}{l}\text { 5. Les dieux nous ont } \\
\text { donné les sens pour } \\
\text { notre utilité }(4)\end{array}$ & $\begin{array}{l}\text { I } 4,5 ; \mathrm{IV} \\
3,11\end{array}$ & $\begin{array}{l}\text { Timée } \\
47 \mathrm{a}-\mathrm{c} \\
\text { (la vue et } \\
\text { l'ouie) }\end{array}$ & ND II $140-142$ \\
\hline 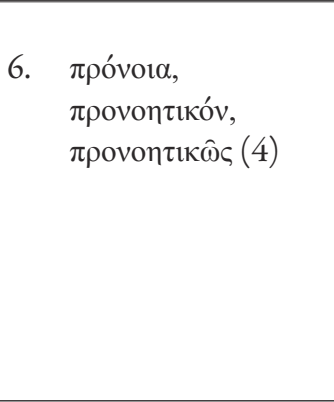 & $\begin{array}{c}\text { I } 4,6 ; \mathrm{IV} \\
3,6\end{array}$ & $\begin{array}{l}\text { Timée 30c, } \\
\text { 44c, } 45 \mathrm{~b} ; \\
\text { aucune } \\
\text { occurrence } \\
\text { pertinente } \\
\text { dans les } \\
\text { Lois }\end{array}$ & 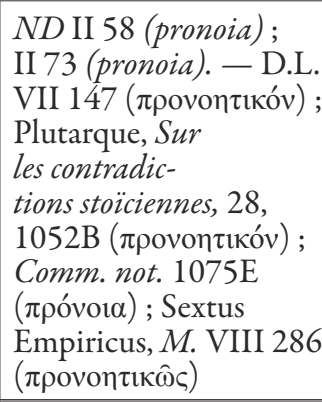 \\
\hline $\begin{array}{l}\text { 7. Les œuvres de la } \\
\text { providence }\end{array}$ & $\begin{array}{c}\text { I } 4,6 \\
(\pi \rho \text { ovo í } \alpha \varsigma \\
\varepsilon \neq \rho \gamma \alpha)\end{array}$ & $\varnothing$ & $\begin{array}{l}\text { ND II } 128 \\
\text { (opera providae... } \\
\text { naturae) }\end{array}$ \\
\hline
\end{tabular}

34. Les testimonia qui figurent dans l'apparat de l'édition Bandini-Dorion sont en caractères gras. Je me suis efforcé de signaler tous les passages du De natura deorum qui font écho au texte des Mémorables. En plus des passages du De natura deorum, j’ai signalé, mais sans aucunement prétendre à l'exhaustivité, des textes stoïciens ou d'inspiration stö̈cienne qui offrent des recoupements avec les Mémorables. Pour la signification des chiffres entre parenthèses, dans la première colonne, voir infra, p. 194 n. 40. 


\begin{tabular}{|c|c|c|c|c|}
\hline & Les paupières (4) & I 4, 6 & $\begin{array}{l}\text { Timée } \\
45 \mathrm{~d}-\mathrm{e}\end{array}$ & ND II 142 \\
\hline & $\begin{array}{l}\text { Les cils } \\
(\beta \lambda \varepsilon \varphi \alpha \rho i ́ \delta \varepsilon \varsigma)(4)\end{array}$ & I 4,6 & $\varnothing$ & ND II 143 \\
\hline 10. & $\begin{array}{l}\text { Les sourcils (ỏ } \varphi \rho \hat{c} \varsigma) \\
\text { arrêtent la sueur qui } \\
\text { coule du front }(4)\end{array}$ & I 4,6 & $\varnothing$ & ND II 143 \\
\hline 11. & $\begin{array}{l}\text { L'oreille est } \\
\text { toujours ouverte } \\
\text { pour percevoir tous } \\
\text { les sons }(4)\end{array}$ & I 4,6 & $\varnothing$ & ND II 144 \\
\hline 12. & 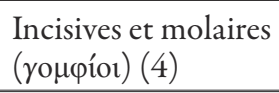 & I 4,6 & $\varnothing$ & ND II 134 \\
\hline 13. & $\begin{array}{l}\text { Évacuation des } \\
\text { excréments loin des } \\
\text { sens (4) }\end{array}$ & I 4,6 & $\varnothing$ & ND II 141 \\
\hline 14. & $\begin{array}{l}\text { Dieu comparé } \\
\text { à un démiurge } \\
(\delta \eta \mu 10 v \rho \gamma \text { ć) })(1) \\
\end{array}$ & I 4,7 & $\begin{array}{c}\text { Timée 28a, } \\
\text { 29a, 41a }\end{array}$ & $\begin{array}{l}\text { ND II } 57-58- \\
\text { D.L. VII } 147\end{array}$ \\
\hline 15 . & $\begin{array}{l}\text { Dieu est } \varphi 1 \lambda o ́ \zeta \omega \circ \varsigma \\
(4)\end{array}$ & I 4,7 & $\varnothing$ & Épictète, fr. 23 \\
\hline 16 . & $\begin{array}{l}\text { Désir de se } \\
\text { reproduire }(4)\end{array}$ & $\mathrm{I} 4,7$ & $\varnothing$ & $\begin{array}{l}\text { ND II } 128 \text { - Épictète, } \\
\text { I } 6,9\end{array}$ \\
\hline 17. & $\begin{array}{l}\text { L'homme a une } \\
\text { parcelle de tous les } \\
\text { éléments }(4)\end{array}$ & I 4,8 & $\begin{array}{l}\text { Philèbe } \\
\text { 29a-30d }\end{array}$ & $\begin{array}{l}\text { ND II 18; II } 37- \\
\text { Marc-Aurèle, IV 4, } 3 ; \\
\text { Philon, Prov. I 4, I 25, } \\
\text { I } 68\end{array}$ \\
\hline 18. & $\begin{array}{l}\text { D'où l'homme tire- } \\
\text { t-il son intelligence? } \\
\text { (1) }\end{array}$ & I 4,8 & $\varnothing$ & ND II 18; II 79; III 27 \\
\hline 19. & $\begin{array}{l}\text { Arrogance de } \\
\text { celui qui considère } \\
\text { que l'homme a } \\
\text { « confisqué » } \\
\text { l'intelligence (1) }\end{array}$ & I 4,8 & $\varnothing$ & $\begin{array}{l}\text { ND II } 16 \text {; II } 44 ; \text { II } 56 . \\
\text { - Philon, Prov. I } 28\end{array}$ \\
\hline 20. & $\begin{array}{l}\text { Le bel ordon- } \\
\text { nancement du } \\
\text { tout dépend de } \\
\text { l'intervention } \\
\text { d'une intelligence } \\
(1,3)\end{array}$ & I 4,8 & $\varnothing$ & $\begin{array}{l}\text { ND II } 15 ; \text { II } 54 ; \text { II } 56 \text {; } \\
\text { II } 90\end{array}$ \\
\hline
\end{tabular}




\begin{tabular}{|c|c|c|c|}
\hline $\begin{array}{l}\text { 21. Dieu ordonne et } \\
\text { maintient tout } \\
\text { l'univers (3) }\end{array}$ & $\begin{array}{l}\text { I } 4,8 ; 17 \\
\text { IV } 3,13\end{array}$ & $\begin{array}{l}\text { Lois X } \\
905 \mathrm{e}\end{array}$ & $\begin{array}{l}\text { ND II } 4 \text {; II 29-30; } \\
\text { II } 44 ; \text { II } 54 ; \text { II } 75-80 \text {; } \\
\text { II } 82 ; \text { II } 85 ; \text { II } 97 ; \\
\text { II } 132\end{array}$ \\
\hline $\begin{array}{l}\text { 22. Les dieux se } \\
\text { soucient des } \\
\text { hommes (4) } \\
\end{array}$ & $\begin{array}{l}\text { I } 4,11 ; 14 ; \\
\quad \text { IV } 3,12\end{array}$ & $\begin{array}{l}\text { Lois X } \\
905 \mathrm{~d}\end{array}$ & $\begin{array}{l}\text { ND II } 133 ; \text { II } 162 \text {; } \\
\text { II } 164-166\end{array}$ \\
\hline $\begin{array}{l}\text { 23. Les dieux ont } \\
\text { privilégié l'homme } \\
\text { par rapport aux } \\
\text { animaux; téléologie } \\
\text { anthropocentrique } \\
\text { (4) }\end{array}$ & $\begin{array}{l}\text { I } 4,11-14 \\
\text { IV } 3,9-10\end{array}$ & $\varnothing$ & $\begin{array}{l}\text { ND II } 133 \text {; II } \\
145-146 \text {; II } 153- \\
\text { Épictète, I 6, 12-22 }\end{array}$ \\
\hline $\begin{array}{l}\text { 24. La station droite } \\
(4)\end{array}$ & I 4,11 & Timée 90b & ND II 140 \\
\hline 25. Les sens (4) & I 4,11 & $\varnothing$ & ND II 141-142 \\
\hline $\begin{array}{l}\text { 26. Les mains et la pros- } \\
\text { périté de l'homme } \\
(4)\end{array}$ & I 4,11 & $\varnothing$ & ND II 150-151 \\
\hline $\begin{array}{l}\text { 27. Éloge de la parole } \\
(4)\end{array}$ & $\begin{array}{c}\text { I } 4,12 ; \text { IV } \\
3,12 \\
\end{array}$ & $\varnothing$ & ND II 148 \\
\hline $\begin{array}{l}\text { 28. La langue et la voix } \\
(4)\end{array}$ & I 4,12 & $\varnothing$ & ND II 149 \\
\hline $\begin{array}{l}\text { 29. L'homme est le } \\
\text { seul être vivant } \\
\text { à reconnaître } \\
\text { l'existence des dieux }\end{array}$ & I 4,13 & $\varnothing$ & ND II 153 \\
\hline $\begin{array}{l}\text { 30. La providence } \\
\text { divine veille sur les } \\
\text { individus }\end{array}$ & I 4,14 & $\begin{array}{l}\text { Contra, } \\
\text { cf. Lois X } \\
903 \mathrm{c}\end{array}$ & ND II 164 \\
\hline $\begin{array}{l}\text { 31. La divination et la } \\
\text { bienveillance divine } \\
\text { (4) }\end{array}$ & $\begin{array}{c}\text { I } 4,15 ; \text { IV } \\
3,12\end{array}$ & Timée 71e & ND II 162-167 \\
\hline $\begin{array}{l}\text { 32. Consensus omnium } \\
\text { gentium (1) }\end{array}$ & I 4,16 & $\begin{array}{c}\text { Lois X } \\
886 \mathrm{a}\end{array}$ & $\begin{array}{l}\text { ND I } 1 ; \text { I } 11 ; \text { II } 5 \text {; } \\
\text { II } 12 ; \text { II } 81\end{array}$ \\
\hline $\begin{array}{l}\text { 33. La divinité est } \\
\text { omniprésente et } \\
\text { toute-puissante (2) }\end{array}$ & I $4,17-18$ & $\varnothing$ & ND II 4 \\
\hline
\end{tabular}




\begin{tabular}{|c|c|c|c|c|}
\hline & $\begin{array}{l}\text { Dieu a créé la } \\
\text { lumière }(4)\end{array}$ & IV 3,3 & $\begin{array}{c}\text { Timée 39b, } \\
45 \mathrm{~b}\end{array}$ & Épictète, I 6, 5 \\
\hline 35. & $\begin{array}{l}\text { Dieu pourvoit à } \\
\text { tous les besoins de } \\
\text { l'homme }(4)\end{array}$ & IV 3, 3-10 & $\varnothing$ & ND II 58 \\
\hline 36. & $\begin{array}{l}\text { La nuit a été conçue } \\
\text { afin de permettre le } \\
\text { repos }(4)\end{array}$ & IV 3,3 & $\varnothing$ & ND II 132 \\
\hline 37. & $\begin{array}{l}\text { Dieu est } \\
\varphi \uparrow \lambda \alpha \dot{v} \theta \rho \omega \pi \mathrm{\alpha}(4)\end{array}$ & IV 3,$5 ; 7$ & $\begin{array}{l}\text { IV } 3,5 ; 7 \\
\text { Lois IV } \\
\text { 713d }\end{array}$ & $\begin{array}{l}\text { ND II } 60 \text { - Plutarque, } \\
\text { Comm. not. } 1075 \mathrm{E}\end{array}$ \\
\hline 38. & $\begin{array}{l}\text { Éloignement et } \\
\text { rapprochement du } \\
\text { soleil }(4)\end{array}$ & IV 3, 8-9 & $\varnothing$ & $\begin{array}{l}\text { ND II } 19 \text {; II } 49 \text {; II } 92 \text {; } \\
\text { II } 102 \text { - Philon, Prov. } \\
\text { II } 78\end{array}$ \\
\hline 39. & 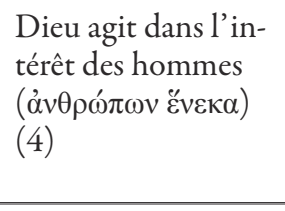 & IV 3,$8 ; 10$ & $\varnothing$ & $\begin{array}{l}\text { hominum causa: ND II } \\
37, \text { II } 133 \text {; II } 154-155 ; \\
\text { II } 158-\text { bominum } \\
\text { gratia: ND II } 158- \\
\text { Philon, Prov. II } 84 \\
\end{array}$ \\
\hline 40. & $\begin{array}{l}\text { Les animaux sont } \\
\text { dans l'intérêt de } \\
\text { l'homme }(4)\end{array}$ & IV 3,10 & $\varnothing$ & ND II 37 ; II 158-162 \\
\hline 41. & $\begin{array}{l}\text { Dieu et l'âme se } \\
\text { rendent visibles par } \\
\text { leurs œuvres (2) }\end{array}$ & IV $3,13-14$ & $\begin{array}{l}\text { Lois X } \\
\text { 898d-e }\end{array}$ & $\begin{array}{l}\text { Tusc. I 69-70-- } \\
\text { Épictète, I 6, } 7 \text {; } \\
\text { Marc-Aurèle XII } 28\end{array}$ \\
\hline 42. & $\begin{array}{l}\text { Distinction entre } \\
\text { un dieu supé- } \\
\text { rieur et des dieux } \\
\text { subordonnés }(2)\end{array}$ & IV 3,13 & Timée 69c & ND I 31 \\
\hline 43. & $\begin{array}{l}\text { Divinité de l'âme } \\
(4)\end{array}$ & IV 3,14 & $\begin{array}{l}\text { Timée } \\
\text { 45a ; Alc. } \\
\text { 1, 133c; } \\
\text { Phédon } \\
\text { 79e-80a }\end{array}$ & $\begin{array}{l}\text { ND II } 34 ; \text { II } 147 \text {; II } \\
154\end{array}$ \\
\hline 44. & $\begin{array}{l}\text { Sacrifier selon ses } \\
\text { moyens }\end{array}$ & IV $3,16-17$ & $\varnothing$ & Épictète, Manuel, 31.5 \\
\hline
\end{tabular}

Afin de bien mettre en évidence l'étroitesse des recoupements entre les Mémorables et le livre II du De natura deorum, j'ai intégré au tableau des passages des dialogues de Platon où l'on constate, ou non, les mêmes recoupements. On pourrait en effet m'objecter que la téléologie stoïcienne ne 
s'inspire pas moins de Platon que de Xénophon, de sorte que mon tableau des recoupements serait à la fois abusif et incomplet, en ce qu'il laisserait faussement entendre que les Mémorables est le seul texte « socratique » qui présenterait de tels recoupements avec le De natura deorum. En cherchant à mettre en lumière l'influence des Mémorables sur le De natura deorum, mon intention n'est pas d'oblitérer ni même de réviser à la baisse l'influence de Platon sur Cicéron ou sur la théologie stoïcienne en général ${ }^{35}$. Le fait même que Balbus, au livre II, qualifie Platon de « dieu des philosophes » (deum philosophorum, II 32) est un indice, parmi d'autres, de la dette que Cicéron se reconnaît à l'endroit de Platon. Pour bien identifier quels sont les recoupements avec les Mémorables qui sont exclusifs aux Mémorables, il est nécessaire de signaler également, le cas échéant, les recoupements avec Platon sur les mêmes thèmes. En introduisant dans ce tableau les recoupements pertinents que l'on trouve - ou non - chez Platon, la « filiation » entre les Mémorables et le De natura deorum n'en ressort que plus clairement dans la mesure où l'on constate que dans plusieurs cas les dialogues de Platon n'offrent pas de recoupement. Les cas d'absence de recoupements avec le Timée sont particulièrement révélateurs si l'on considère que Cicéron a traduit ce dialogue ${ }^{36}$. Et même lorsqu'il y a un recoupement avec un texte platonicien, on peut aisément démontrer, dans certains cas, que Platon n'est pas la source de Cicéron. J'en fournirai plus tard deux exemples ${ }^{37}$.

Étant donné qu'il est impossible de commenter tous les recoupements signalés dans ce tableau, je m'en tiendrai aux observations qui me paraissent les plus importantes.

Krohn a déjà proposé un certain nombre de rapprochements entre les Mémorables et le livre II du De natura deorum ${ }^{38}$, mais son objectif, en dressant la liste des passages parallèles, n'était pas de mettre en lumière l'influence des Mémorables sur la théologie stoïcienne, mais au contraire de « démontrer » que les passages parallèles, en Mem. I 4 et IV 3, étaient à l'évidence des interpolations d'origine stoïcienne.

Alors que l'apparat des testimonia de l'édition Bandini-Dorion recense uniquement trois passages des Mémorables (I 4, 5-6 ; I 4, 8 ; IV 3, 13) pour lesquels on trouve des échos dans le De natura deorum, ce tableau en identifie une quarantaine, ce qui est un enrichissement considérable des testimonia pour Mem. I 4 et IV 3.

Il y a un important recoupement qui ne figure pas dans le tableau car il concerne, non pas un passage ou un argument en particulier, mais la structure même de l'argumentation développée dans les Mémorables et le De natura

35. Sur les origines platoniciennes de la théologie stoïcienne, $c f$. Ademollo 2012.

36. Sur les rapports de Cicéron au Timée, cf. en dernier lieu Reydams-Schils 2013.

37. Voir plus bas la discussion des thèmes $n^{\circ} 8$ et 24 du Tableau.

38. Cf. Krohn 1875 , p. $12,13,15$ et 55 . 
deorum. Au début du livre II du De natura deorum, le stoïcien Balbus affirme ce qui suit :

Nos auteurs, d'une manière générale, divisent en quatre parties l'ensemble de cette recherche sur les dieux immortels : ils enseignent d'abord que les dieux existent, puis quelle est leur nature, ensuite que le monde est gouverné par eux, enfin qu'ils veillent sur les affaires humaines ${ }^{39}$.

Bien que Xénophon ou Socrate ne les identifient pas expressément, ces quatre parties sont également présentes dans les Mémorables ${ }^{40}$. Cette homologie argumentative n'est probablement pas accidentelle, ainsi que le suggère une comparaison avec le livre X des Lois, où l'on ne trouve pas la même structure argumentative ${ }^{41}$. Dans les Lois, en effet, l'Athénien cherche à démontrer trois choses $\left.{ }^{42}: 1\right)$ que les dieux existent $(885 \mathrm{~b}-899 \mathrm{~d})$; 2) qu' ils se soucient des hommes (899d-905d) ; 3) que l'on ne peut pas les fléchir par des prières ou des sacrifices (905d-907b). Le troisième point du programme du livre $\mathrm{X}$, à savoir que les dieux sont inflexibles, est absent aussi bien des Mémorables que du livre II du De natura deorum. Les deux premiers points sont présents dans les deux autres textes, mais l'argumentation développée par Platon pour démontrer l'existence des dieux n'est pas du tout la même que dans les Mémorables et le De natura deorum. En ce qui concerne le deuxième point, à savoir que les dieux veillent sur les hommes, il est certes affirmé par l'Étranger ( $c f$. X 905d), mais ce dernier se soucie beaucoup plus de démontrer que les dieux veillent sur toutes choses ( $c f$. X 900d, 903b, e, 904a) que sur les hommes en particulier. Ces différences avec les Lois tendent à confirmer l'opinion de Sedley suivant laquelle ce dialogue de Platon « ne [me] semble jouer aucun rôle majeur ou évident dans le stoïcisme, sans aucun doute parce que rien chez Platon ne l'associe à Socrate ${ }^{43}$. $\gg$ Dans ce qui suit, je présenterai des exemples de recoupements empruntés à la démonstration de l'existence des dieux et à la démonstration de leur providence.

La démonstration de l'existence des dieux est fondamentalement la même dans les Mémorables et chez Cicéron ${ }^{44}$. Dans les Mémorables $(\mathrm{I} 4,6)$, Socrate place son interlocuteur devant une alternative : ou bien les hommes sont les fruits du hasard, ou bien ils sont les produits d'une intelligence ( $\pi$ ó $\varepsilon \rho \alpha \tau u ́ \chi \eta \varsigma$

39. Cf. II 3. Tous les extraits du De natura deorum cités dans cette étude sont empruntés à la traduction de C. Auvray-Assayas (2002).

40. Certes, ces quatre parties ne se succèdent pas dans cet ordre dans les deux chapitres des Mémorables, mais l'on peut aisément rattacher chacun des thèmes identifiés dans le tableau à l'une ou l'autre de ces quatre parties. Le chiffre entre parenthèses qui suit l'énoncé du thème indique à quelle partie il se rattache.

41. Je suis donc en désaccord avec Festugière (1949, p. 79), qui estime au contraire que le plan de Mem. I 4 « est le plan même de l'exposé des Lois », à quelques différences près.

42. Cf. X 885b, 888c, 907b.

43. Sedley 2005, p. 469.

44. Voir Tableau, thèmes no 2 et 3 . 


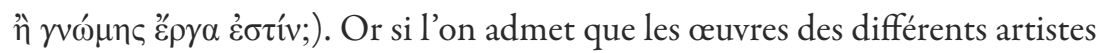
(poètes, peintres, sculpteurs) qui représentent l'homme sont des œuvres qui procèdent d'une intelligence, bien qu'elles soient des images privées de sentiment et de mouvement, on devrait également admettre que l'homme lui-même, qui est animé et en mouvement, est également l'œuvre d'une intelligence. Et comme il ne fait aucun doute que l'homme a été magnifiquement conçu, on devrait également admettre qu'il est l'œuvre d'une intelligence bienveillante à son endroit. D'après Sedley, cet argument constitue « le plus ancien specimen, ou en tout cas le premier ancêtre, de "la preuve par la providence" $\gg{ }^{45}$. Cette démonstration de l'existence du dieu est également présente dans le De natura deorum, où Cicéron développe à plusieurs reprises (II 15, II 87-88, II 97) la même alternative : ou bien le monde est le fruit du hasard, ou bien il est l'œuvre de l'art et d'une intelligence ${ }^{46}$.

Comme on peut le constater en consultant le tableau, la très grande majorité des recoupements concerne le quatrième argument exposé par Balbus (II 3), à savoir que les dieux veillent sur les hommes. C'est une position qui est également affirmée par l'Athénien au livre X des Lois (905d), mais elle n'est pas véritablement développée, c'est-à-dire que l'Athénien n'explique pas vraiment comment ni en quoi les dieux veillent sur les hommes. Le souci que les dieux ont pour les hommes, au livre $\mathrm{X}$ des Lois, ne s'inscrit pas du tout, contrairement aux Mémorables et au De natura deorum, dans une perspective anthropocentrique. Ce que l'on peut sans hésitation nommer l'anthropocentrisme de la théo-téléologie des Mémorables est beaucoup plus développé ${ }^{47}$ que dans les Lois et, comme nous le verrons, il recoupe sur de nombreux points l'anthropocentrisme du livre II du De natura deorum. Mais avant de présenter les principaux recoupements, j'aimerais faire quelques observations sur le vocabulaire de la providence divine.

Un terme-clé pour exprimer le fait que les dieux se soucient des hommes et qu'ils veillent sur eux est le terme $\pi \rho$ óvor $^{48}$, qui correspond au latin

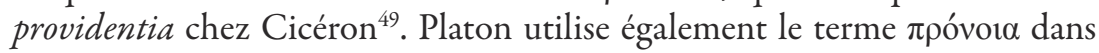

45. Sedley 2005, p. 474. Cf. aussi Sedley 2005, p. 461 et 467 ; Powers 2009, p. 249.

46. Cette preuve de l'existence du dieu n'est pas la seule qui soit développée par Balbus au livre II. Cf. II 12-14 et II 16 pour d'autres preuves de l'existence du dieu.

47. Sedley 2005, p.465: «Socrate développe une téléologie qui est bien plus ouvertement et explicitement anthropocentriste que tout ce que l'on peut trouver chez ses prédécesseurs. » Voir aussi Viano 2001, p. 117 : « La caractéristique la plus frappante de cette vision cosmologique des Mémorables est l'anthropocentrisme : le monde entier est fabriqué et réglé par la divinité en vue de la plus grande utilité pour l'homme. »

48. Voir Tableau, thème $\mathrm{n}^{\circ} 6$.

49. Cf. II 58 : « Telle est donc l'intelligence du monde, que pour cette raison on peut légitimement appeler sagesse ou providence (en grec pronoia) [...] ». II 73 : «C'est ainsi qu'hier tu as dit que les stoïciens mettent en scène une vieille qui prophétise, Pronoia, c'està-dire Providence. » 


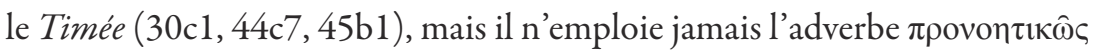

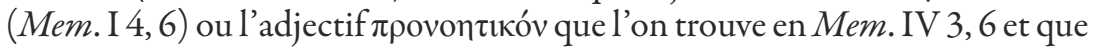
l'on rencontre également au livre VII de Diogène Laërce ${ }^{50}$ et chez Plutarque ${ }^{51}$. Enfin, l'expression opera providae ... naturae, que Cicéron emploie en II 128, rappelle l'expression presque identique ( $\pi \rho$ ovoí $\alpha \varsigma$ है $\rho \gamma \alpha)$ que l'on trouve sous la plume de Xénophon en Mem. I 4, 6.

Un autre terme que Xénophon emploie pour exprimer la bienveillance des dieux à l'endroit des animaux en général est l'adjectif $\varphi \imath \lambda o ́ \zeta \omega o c^{52}$. C'est un terme qui est propre à Xénophon car on n'en relève aucune occurrence chez Platon. On trouve également ce terme dans un fragment d'Épictète qui

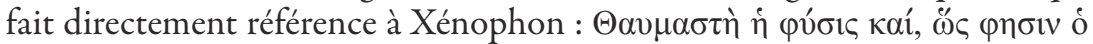
$\Xi \varepsilon v 0 \varphi \hat{\omega} v, \varphi \imath \lambda o ́ \zeta \omega \circ \varsigma$ (fr. 23). Mais contrairement à ce que prétend Épictète, ce n'est pas à la nature que Xénophon reconnaît la qualité d'être $\varphi \imath \lambda o ́ \zeta \omega o c$. Je commenterai en conclusion l'importance de cette différence et de ce déplacement de la divinité à la nature.

En Mem. IV 3, Xénophon emploie deux fois l'expression ảv $\theta \rho \omega ́ \pi \omega v ~ \varepsilon ̌ v \varepsilon \kappa \alpha$ pour exprimer la perspective anthropocentrique de l'organisation divine

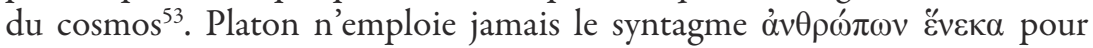
exprimer la perspective anthropocentrique de l'univers conçu par les dieux ${ }^{54}$. Or Cicéron emploie à plusieurs reprises les expressions hominum causa ${ }^{55}$ et hominum gratia ${ }^{56}$, qui sont des expressions rigoureusement équivalentes à

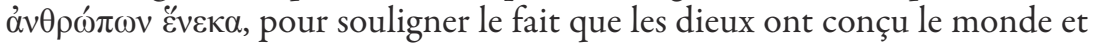
la nature en vue des hommes, et dans leur meilleur intérêt ${ }^{57}$.

50. Cf. VII 147 : « Dieu est un vivant immortel, rationnel, parfait ou bien (un être) intelligent vivant dans la béatitude, ne pouvant recevoir en lui rien de mauvais, exerçant une provi-

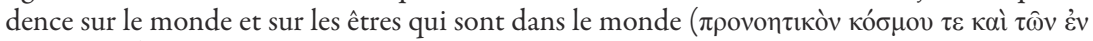

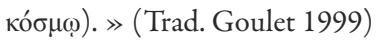

51. Sur les contradictions stö̈ciennes, 38, 1052B.

52. Voir Tableau, thème $\mathrm{n}^{\circ} 15$.

53. Voir Tableau, thème $\mathrm{n}^{\circ}$ 39. Dans le chapitre qui suit Mem. IV 3, Socrate affirme pareillement que les dieux ont établi les lois non écrites dans l'intérêt des hommes ( $\theta \varepsilon$ cò

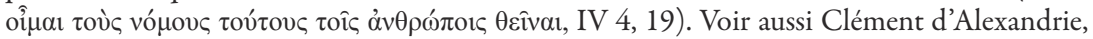

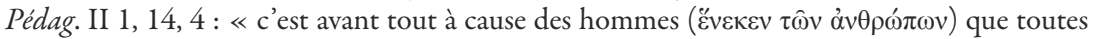
choses ont été créées 》 (trad. Mondésert 1965).

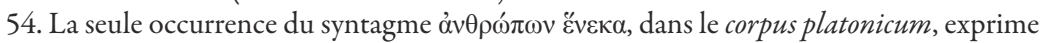
en faveur de qui les lois - et non pas l'univers ou la nature - ont été conçues : « Les lois sont

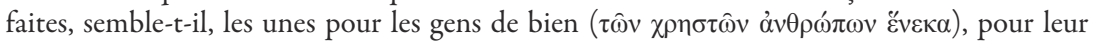
enseigner comment se comporter les uns avec les autres s'ils veulent vivre en paix et en bienveillance mutuelle » (Lois IX 880d; trad. Diès 1956).

55. Cf. II 37, II 133 ; II 154-155; II 158.

56. II 158. Cicéron emploie également les expressions ad hominum commoditates (II 158) et ad usum hominum (II 162).

57. Cf. entre autres, ND II 154 : « Reste à montrer, pour conclure enfin, que tout ce qui existe dans le monde et dont les hommes font usage a été fait et disposé pour les hommes (bominum causa). » 
La perspective anthropocentrique qui est développée par Socrate dans les Mémorables peut être analysée en six étapes qui s'articulent étroitement l'une à l'autre : 1) Tout ce qui a été conçu par les dieux dans l'univers l'a été dans l'intérêt des hommes (IV 3, 3-8). 2) Or une objection vient immédiatement à l'esprit : les bonnes choses conçues par les dieux, par exemple la lumière, la nuit pour se reposer, les bienfaits du soleil, etc., ne profitent-elles pas également aux autres animaux (IV 3,9) ? 3) La réponse à cette objection consiste à dire que les dieux ont favorisé les hommes par rapport aux autres animaux (IV 3, 10-12) ${ }^{58}$. Cette troisième étape se subdivise elle-même en trois moments : 4) premièrement, les animaux eux-mêmes ont été conçus en vue des hommes (IV 3, 10); 5) deuxièmement, le corps de l'homme est supérieur à celui des autres animaux $(\mathrm{I} 4,11-12)$; 6) troisièmement, l'âme humaine est supérieure à celle des autres animaux, puisque l'homme est le seul animal à posséder la raison, qui l'apparente aux dieux (I 4, 13-14).

Chacune des six étapes que l'on vient d'identifier dans le texte des Mémorables est également présente chez Cicéron. Voyons cela plus en détail. 1) J'ai

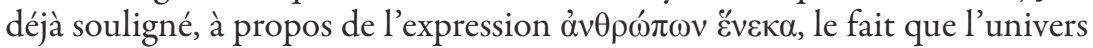
a été conçu dans le meilleur intérêt des hommes. Or l'on trouve, dans l'argumentation déployée par Xénophon et Cicéron pour justifier cette perspective anthropocentrique, un parallèle très étroit qui mérite d'être souligné et qui concerne la course du soleil et les saisons ${ }^{59}$ :

Et le soleil, après qu'il a rebroussé chemin en hiver, s'approche et fait mûrir certains fruits, en dessèche d'autres, chacun à sa saison, et, cela accompli, il cesse de s'approcher et se retire pour se garder de nous nuire en chauffant plus qu'il n'est nécessaire. Et lorsque, après s'être éloigné de nouveau, il parvient à l'endroit où il est pour nous évident que, s'il continuait de s'éloigner, nous serions congelés sous l'effet du froid, il tourne à nouveau, s'avance et revient précisément à l'endroit du ciel où il nous est le plus utile. - Par Zeus, répondit-il, cela aussi semble bien se produire dans l'intérêt des hommes ( $\dot{\alpha} \theta \rho \omega ́ \pi \omega v ~ \varepsilon ̋ v \varepsilon \kappa \alpha)$. [9] - Comme il est également clair que nous ne pourrions supporter ni la chaleur ardente ni le froid, s'ils survenaient tout à coup, c'est si progressivement que le soleil s'approche, et si progressivement qu'il

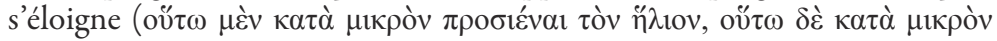
$\dot{\alpha} \pi(\varepsilon ́ v a l)$, que nous arrivons aux températures extrêmes sans même nous en apercevoir. - Pour ma part, répondit Euthydème, j'en suis déjà à examiner si par hasard les dieux ont une autre fonction que de prendre soin des hommes

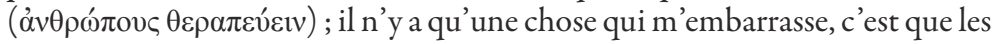
autres animaux ont également part à ces avantages (Mem. IV 3, 8-9).

Ce sont les approches et les éloignements modérés du soleil (solis tum accessus modici tum recessus), encore, qui règlent la distribution du froid et du chaud.

58. Sur la supériorité de l'homme sur les autres animaux, $c f$. aussi $C y r$. VIII 3, 49.

59. Voir Tableau, thème no 38. 
Car le circuit de trois cent soixante-cinq révolutions du soleil et le quart d'un jour environ constituent sa révolution annuelle. Le soleil, infléchissant sa course tantôt vers le septentrion, tantôt vers le midi, produit les étés et les hivers, ainsi que les deux saisons dont l'une s'ajoute à l'hiver finissant et l'autre à l'été ; ainsi, des changements des quatre saisons dérivent l'origine et la cause de tous les êtres qui viennent au monde sur terre et sur mer (omnium quae terra marique gignuntur). (ND II 49)

La pertinence du rapprochement entre ces deux textes, où l'on trouve le même lien entre le cycle des saisons d'une part, et l'alternance de l'éloignement et du rapprochement du soleil d'autre part, ne fait guère de doute. Et c'est presque dans les mêmes termes que Xénophon et Cicéron soulignent qu'il s'agit d'un éloignement et d'un rapprochement graduels et progressifs. Il y a néanmoins une différence qui mérite d'être soulignée. La perspective de Socrate, dans les Mémorables, est résolument anthropocentrique : c'est dans l'intérêt des hommes que le soleil s'éloigne et se rapproche et qu'il est ainsi à l'origine des saisons. Le passage se termine sur une objection d'Euthydème : mais cela ne profite-t-il pas à l'ensemble des animaux ? Ce qui correspond à l'objection que j'ai identifiée comme la deuxième étape (2) de l'argumentation anthropocentrique. Or le passage correspondant de Cicéron n'est pas anthropocentrique puisque la fin du passage cité affirme clairement que les mouvements du soleil profitent à l'ensemble des êtres vivants. Tout se passe comme si le texte de Cicéron avait intégré l'objection d'Euthydème à son argumentation.

3) La troisième étape, qui correspond à la réponse de Socrate à l'objection d'Euthydème, consiste à affirmer que les dieux ont favorisé les hommes par rapport aux autres animaux, sous le double rapport du corps et de l'âme (Mem. I 4, 11-13 ; IV 3, 11-12). Cicéron, de même, affirme clairement la supériorité de l'homme sur les autres animaux : «Par cet exposé, je crois avoir suffisamment montré combien la nature humaine l'emporte sur tous les autres êtres vivants » (II 153). Cette troisième étape se subdivise en trois moments. 4) Premièrement, les deux auteurs affirment clairement que les animaux existent dans l'intérêt des hommes ${ }^{60}$ :

Mais n'est-il pas aussi évident, demanda Socrate, que c'est également dans

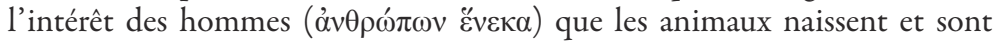
élevés? Quel autre animal retire autant de biens que l'homme des chèvres, des moutons, des bœufs, des chevaux, des ânes et des autres animaux ? J'ai l'impression qu'il en retire encore plus que des plantes : celles-ci ne donnent pas moins de nourriture et de richesse que ceux-là, mais une grande partie du genre humain ne se nourrit pas de ce que la terre produit, mais vit du lait, du

60. Voir Tableau, thème $\mathrm{n}^{\circ}$ 40. Selon Powers (2009, p. 261), « this [scil. l'affirmation que les animaux existent pour le profit de l'homme] appears to be the earliest explicit Greek formulation of this influential and long-lived view ». 
fromage et de la viande que leur fournit le bétail. Or tous les hommes apprivoisent et domptent les animaux utiles et ils s'en servent comme auxiliaires à la guerre et pour une foule d'autres tâches. - Je suis également de ton avis sur ce point, répondit-il. Je vois en effet que des animaux beaucoup plus forts que nous sont devenus tellement soumis à l'homme qu' il peut les utiliser à ce qu'il veut. (Mem. IV 3, 10)

Il faut donc admettre que cette abondance de biens est destinée à l'homme (hominum ... causa). À moins peut-être que la profusion et la variété de fruits, à moins que l'agrément qu'on trouve non seulement à les goûter mais même à les sentir et à les voir ne fassent douter que la nature ait réservé ces présents aux hommes! Les bêtes, loin d'être les destinataires de ces présents, ont été elles-mêmes créées pour les hommes (hominum gratia), nous le voyons : à quoi servent en effet les brebis sinon à vêtir les hommes de leur laine, une fois qu'on l'a travaillée et tissée? À vrai dire, elles auraient été incapables de se nourrir, de se sustenter et de produire quoi que ce soit sans les soins apportés par les hommes à leur entretien. Et les chiens ? Leur garde si fidèle, leurs caresses si aimantes pour leurs maîtres, leur haine si vive des étrangers, leur flair incroyable quand ils suivent une piste, leur ardeur à la chasse, que signifie tout cela, sinon qu'ils ont été créés dans l'intérêt des hommes (ad hominum commodidates) ? (ND II 158)

On trouve dans ce texte de Cicéron la même progression que chez Xénophon : Balbus commence par affirmer que tous les biens existent dans l'intérêt des hommes; puis il évoque la possibilité que ces biens ne s'adressent pas moins aux autres êtres vivants; enfin, il justifie sa première affirmation en soutenant que les animaux font eux-mêmes partie des biens qui existent dans l'intérêt des hommes ${ }^{61}$. 5) Deuxièmement, les deux auteurs affirment que le corps de l'homme est supérieur à celui des animaux. Parmi les avantages du corps humain sur celui des animaux, ils mentionnent l'un et l'autre la station droite $^{62}$ et les sens ${ }^{63}$.

61. Voir aussi Aristote, Pol. I 8, 1256 b 15-22 : « Dès lors il faut évidemment croire que la nature subvient de même aux adultes et que les plantes sont faites pour les animaux et les

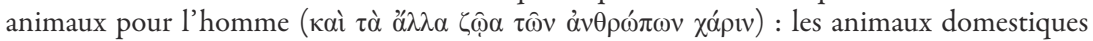
servent à son usage et à sa nourriture ; les animaux sauvages, sinon tous, du moins la plupart, servent à sa nourriture et à ses autres besoins, pour qu'il en tire soit son habillement, soit divers instruments. Si donc la nature ne fait rien sans but ni en vain, il faut admettre que c'est

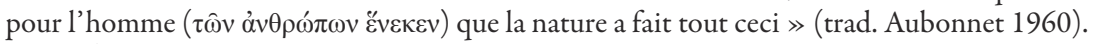
Sedley (2008, p. 331-332) considère qu'Aristote est ici tributaire du Socrate de Xénophon.

62. Voir Tableau, thème no 24. Cf. aussi Auvray-Assayas 2002, p. 120 n. 128 (ad II 140) : «Sur la position debout, propre à l'homme, voir Leg. 1, 26 (et le texte de Xénophon, Mem. 14,11, souvent donné comme source). »

63. Voir Tableau, thème no 25. Cf. aussi Auvray-Assayas 2002, p. 120 n. 129 (ad II 141) : «La description des organes sensoriels peut être comparée aux textes des Mémorables $(14,6)$ et du Banquet $(5,6)$ de Xénophon. » Le passage du Banquet (V 6) ne vise pas à établir la supériorité des organes sensoriels de l'homme sur ceux des animaux, mais plutôt à établir que le nez de Socrate est plus beau que celui de Critobule pour autant qu'il est mieux adapté à sa 
[Aristodème] Sache bien, dit-il, que si je croyais que les dieux ont quelque

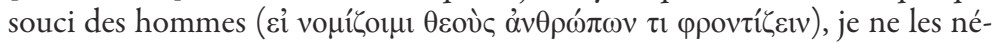
gligerais pas. - [Socrate] Alors tu ne crois pas qu'ils s'en soucient ? Eux qui, pour commencer, ont fait de l'homme le seul des animaux à se tenir debout ! Or c'est la station droite qui lui permet de porter le regard plus loin, de mieux observer ce qui est au-dessus de lui, et d'être moins exposé au danger ; aussi ont-ils placé en haut la vue, l'ouie et le goût. (Mem. I 4, 11)

On peut donner beaucoup d'autres exemples de cette providence de la nature (providentiam naturae), si diligente et si adroite, pour faire comprendre l'importance et l'excellence des dons que les dieux ont faits aux hommes. D'abord la divinité les a dressés debout sur le sol, la taille haute et droite, pour leur permettre, en regardant le ciel, d'acquérir la connaissance des dieux. Sur la terre, les hommes ne sont pas des indigènes ou des habitants, mais pour ainsi dire des spectateurs des réalités supérieures du ciel, dont le spectacle ne touche aucune espèce d'êtres vivants. Les sens, interprètes et messagers des choses, situés dans la tête comme une citadelle, sont admirablement adaptés, à la fois par leur structure et par leur position, à des fonctions indispensables : les yeux, comme des guetteurs, occupent l'endroit le plus élevé, d'où ils peuvent s'acquitter de leur tâche, en inspectant une très vaste étendue. Les oreilles aussi, qui doivent percevoir le son, qui se porte naturellement vers le haut, sont placées comme il convient dans la partie supérieure du corps. De même, le nez est en haut, comme il convient, parce que les odeurs vont toujours en montant; d'autre part, comme il joue un grand rôle dans l'appréciation de la nourriture et de la boisson, ce n'est pas sans raison qu'il a cherché le voisinage de la bouche. Quant au goût qui doit tester les saveurs de nos divers aliments, il a son siège dans la partie de la bouche où la nature a ouvert le chemin à ce qui se mange et à ce qui se boit. (ND II 140-141)

Platon fait également état, dans le Timée (90b), de la station droite dont les dieux ont gratifié l'homme, mais ce passage du Timée n'est probablement pas la source de Cicéron. Dans le texte de Xénophon, la station droite est immédiatement suivie de la mention des sens qui logent dans la tête (la vue, l'ouie et le goût). Or on observe exactement la même séquence chez Cicéron (vue, ouie, goût), à cette différence qu'il ajoute l'odorat, complétant ainsi ce qui a tout l'air d'un oubli dans le texte de Xénophon. Chez Platon, la mention de la station droite n'est pas suivie de l'énumération des sens qui logent dans la tête ${ }^{64}$.

fonction. La dimension théo-téléologique qui sous-tend Mem. I 4 et IV 3 affleure néanmoins dans l'affirmation de Socrate suivant laquelle les dieux ont fabriqué des nez pour permettre aux hommes de sentir (

64. Certes, la métaphore de la citadelle (in arce, II 140 ; voir aussi Tusc. I 20 : in capite sicut in arce) pour désigner la tête provient sans doute d'un passage du Timée où Platon emploie

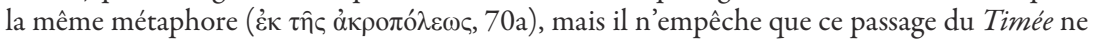
traite ni de la station droite, ni des sens qui logent dans la tête. 
Xénophon et Cicéron complètent leur énumération des avantages du corps humain en mentionnant les mains ${ }^{65}$, la langue ${ }^{66}$ et l'usage de la parole ${ }^{67}$ :

Ensuite, alors qu' ils ont doté les autres animaux terrestres de pieds, qui leur permettent seulement de se déplacer, ils ont de plus pourvu l'homme de mains, qui exécutent la plupart des choses grâce auxquelles nous sommes plus prospères que les animaux. (Mem. I 4, 11)

Quelles servantes habiles, et cela dans combien d'arts, sont les mains que la nature a données à l'homme ! [...] On comprend comment grâce aux mains des artisans qui mettent en œuvre les inventions de l'esprit et les perceptions des sens, nous nous sommes procuré tout ce qu'il nous fallait pour être abrités, vêtus et en sécurité, et nous avons des villes, des murailles, des habitations, des temples. (ND II 150)

Et bien que tous les animaux aient une langue, ils ont conçu celle de l'homme, et elle seule, de telle sorte qu'en touchant la bouche, tantôt ici, tantôt là, elle articule la voix et exprime toutes les choses que nous désirons nous communiquer les uns aux autres. (Mem. I 4, 12)

Ils nous ont également fait don de l'expression, qui nous permet, par l'instruction mutuelle, de nous communiquer tous les biens, de les mettre en commun, d'établir des lois et de nous gouverner. - [Euthydème] Il semble bien, Socrate, que les dieux portent la plus grande attention aux hommes (oi

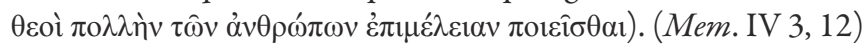

Et que dire de cette reine du monde, comme vous dites, la parole ? Qu'elle est admirable, qu'elle est divine! D'abord, c'est grâce à elle que nous pouvons apprendre ce que nous ignorons et enseigner à d'autres ce que nous savons ; et puis, c'est par elle que nous exhortons, nous persuadons, nous consolons les affligés, nous rassurons ceux qui ont peur, nous calmons les impatients, nous éteignons les passions et les colères ; c'est elle qui nous a liés par la communauté de droit, des lois et des cités, c'est elle qui nous a fait sortir de l'état de barbarie et de sauvagerie. (ND II 149)

À propos des sens, Cicéron affirme très clairement que « les sens des hommes sont bien supérieurs à ceux des animaux » (II 145). Commentant cette affirmation, C. Auvray-Assayas soutient que « l'affirmation de la supériorité sensorielle de l'homme sur les bêtes n'a pas de parallèle connu avant Cicéron ${ }^{68}$. Certes, Socrate n'affirme pas expressément la supériorité sensorielle de l'homme sur les animaux, mais il affirme tout de même que les hommes sont supérieurs aux animaux et du point de vue du corps, et du point de vue de l'âme, de sorte qu'il leur est forcément supérieur du point de vue des sens également.

65. Voir Tableau, thème $\mathrm{n}^{\circ} 26$.

66. Voir Tableau, thème $\mathrm{n}^{\circ} 28$.

67. Voir Tableau, thème $\mathrm{n}^{\circ} 27$.

68. Auvray-Assayas 2002, p. 123 n. 131. 
Ne te saute-t-il pas aux yeux que <les $>$ hommes, en comparaison des autres animaux, vivent comme des dieux, dotés qu'ils sont d'un corps et d'une âme qui sont par nature supérieurs ( $\kappa \rho \alpha \tau \iota \sigma \tau \varepsilon \dot{o v} \tau \varepsilon \varsigma)$ ? Car l'animal qui aurait le corps d'un bœuf et l'intelligence propre à l'homme ne pourrait pas faire ce qu'il voudrait, et tous les animaux qui ont des mains, mais qui sont dépourvus d'esprit, n'ont aucun avantage. Et toi, qui as obtenu les deux choses les plus précieuses ( $\Sigma \grave{v} \delta ’ \grave{\alpha} \mu \varphi \circ \tau \varepsilon^{\prime} \rho \omega v \tau \hat{\omega} v$

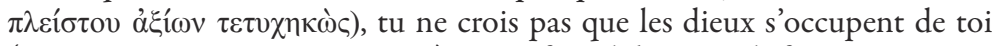

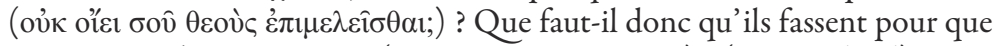

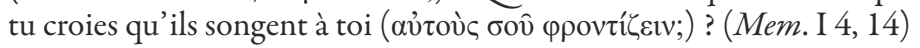

Toujours à propos du corps humain, Socrate explique que les organes et les parties du corps n'ont pas été conçus au hasard, mais qu'ils ont été intentionnellement façonnés de façon à remplir au mieux leur fonction. Il donne plusieurs exemples et la plupart d'entre eux sont également mentionnés par Cicéron dans le De natura deorum. Or ces parallèles évidents ne figurent pas dans l'apparat des testimonia au texte des Mémorables. Commençons par les paupières ${ }^{69}$ :

Par exemple, comme la vue est fragile, on l'a munie de paupières qui, comme des portes, s'ouvrent toutes grandes lorsqu'on a besoin d'utiliser la vue, mais qui se ferment pendant le sommeil. (Mem. I 4, 6)

[...] Quant aux paupières, qui sont les couvertures des yeux, elles sont très douces au toucher pour ne pas blesser la pupille et très bien aménagées pour couvrir les pupilles, afin que rien n'y pénètre, et pour les découvrir; et elle [scil. la nature] a pourvu à ce que cela pût se faire continuellement, avec la plus grande rapidité. (ND II 142)

Platon parle également des paupières qui ont été conçues par les dieux pour protéger l'œil, mais, là encore, ce passage du Timée (45d-e) n'est probablement pas la source de Cicéron. La mention des paupières n'est en effet pas suivie par celle des cils et des sourcils, alors qu'on trouve chez Xénophon et Cicéron la même séquence : paupières, cils, sourcils. En plus des paupières, des cils et des sourcils, Cicéron reprend trois autres exemples qui se trouvent également en Mem. I 4, 6, soit les exemples des oreilles, des molaires et des incisives, et des voies d'évacuation des excréments. Qui plus est, les trois premiers exemples qui suivent immédiatement les paupières (cils, sourcils, oreilles) sont présentés dans le même ordre que dans les Mémorables. D'abord les cils ${ }^{70}$ :

Et pour que même les vents ne puissent nuire à la vue, on a greffé sur les paupières des cils qui tiennent lieu de crible. (Mem. I 4, 6)

Les paupières sont munies d'une espèce de rempart de poils, destinés à repousser, quand les yeux sont ouverts, ce qui pourrait y tomber, et quand ils

69. Voir Tableau, thème $n^{\circ} 8$.

70. Voir Tableau, thème n ${ }^{\circ} 9$. 
sont clos par le sommeil, alors que nous n'en avons pas besoin pour voir, pour leur permettre de se reposer, dans leur enveloppe. (ND II 143)

Ensuite les sourcils ${ }^{71}$ :

Pour que la sueur qui découle de la tête ne gêne pas non plus la vue, on a garni ce qui est au-dessus des yeux de sourcils qui font office de corniche. (Mem. I 4, 6)

La partie supérieure, avec sa couverture de sourcils, arrête la sueur qui coule de la tête et du front. (ND II 143)

Finalement les oreilles ${ }^{72}$ :

Et le fait que l'oreille perçoive tous les sons, sans jamais s'obstruer... (Mem. I 4, 6)

Mais l'organe de l'ouie est toujours ouvert, car même en dormant nous en avons besoin [...] (ND II 144)

Mais aussi les incisives et les molaires ${ }^{73}$ :

Et que, chez tous les animaux, les dents de l'avant soient propres à couper et les molaires à broyer ce qu'elles reçoivent des premières... (Mem. I 4, 6)

On mâche avec les dents disposées dans la bouche; par elles les aliments sont amenuisés et amollis. Celles de devant, les incisives, les réduisent en morceaux, celles du fond, appelées molaires, les mastiquent... (ND II 134)

Et les voies d'évacuation des excréments ${ }^{74}$ :

Et que l'on ait placé la bouche, par laquelle les animaux absorbent ce qu'ils désirent, en bas près des yeux et du nez, alors que, comme les excréments sont désagréables, on en a détourné et écarté les canaux d'évacuation aussi loin que possible des organes sensoriels. (Mem. I 4, 6)

Et, de même que, dans les maisons, les architectes éloignent des yeux et du nez des propriétaires les canaux de vidange qui auraient nécessairement quelque chose de répugnant, de même la nature a relégué loin de nos sens les choses de ce genre. (ND II 141)

6) Troisièmement, les deux auteurs affirment que la raison distingue l'homme de l'animal et que c'est là une preuve additionnelle de la providence divine et de la bienveillance des dieux à l'endroit des hommes ${ }^{75}$.

71. Voir Tableau, thème $n^{\circ} 10$. Selon plusieurs commentateurs ( $c f$. ., entre autres, Sedley 2005, p. 467 n. 14), le passage des Mémorables sur les cils et les sourcils serait la source directe du passage parallèle du De partibus animalium (II 15, 658b14-18).

72. Voir Tableau, thème $\mathrm{n}^{\circ} 11$.

73. Voir Tableau, thème $\mathrm{n}^{\circ} 12$. L'exemple des incisives et des molaires se trouve également chez Aristote (cf. Ph. II 198b24-26 ; PA III 1, 661b6-9) et il n'est pas exclu que le Stagirite soit à nouveau tributaire de Xénophon ( $c f$. Sedley 2005, p. 467 n. 14), de sorte que même si Cicéron empruntait ces exemples à Aristote, leur source indirecte serait néanmoins Xénophon.

74. Voir Tableau, thème $\mathrm{n}^{\circ} 13$.

75. Voir Tableau, thème $n^{\circ} 43$. 
Nous avons effectué un survol des principaux recoupements entre Xénophon et Cicéron pour la première et la quatrième des thèses présentées par Balbus au début du livre II. J'aimerais, avant de conclure, présenter un autre recoupement qui n'est pas moins significatif que les précédents. Il est en effet remarquable que les deux auteurs terminent l'un et l'autre leur exposé sur la théo-téléologie par un développement sur la divination ${ }^{76}$. Dans les Mémorables, le développement sur la divination s'inscrit dans le prolongement de la démonstration de la providence divine à l'endroit des hommes : la preuve ultime que les dieux se soucient des hommes et qu'ils veillent sur eux est précisément qu'ils leur envoient régulièrement des signes sur ce qu'ils doivent entreprendre ou s'abstenir de faire. Le livre II du De natura deorum se termine pareillement par un développement sur la divination et cette conclusion du livre II s'inscrit elle aussi dans le prolongement naturel du long développement sur la providence divine. De plus, c'est également au sein de leur passage respectif sur la divination que les deux auteurs insistent sur le fait que les dieux, dans leur souci providentiel, ne veillent pas moins sur les hommes individuels que sur l'humanité en général ${ }^{77}$.

\section{Conclusion}

La recherche des sources (Quellenforschung) m'a toujours inspiré la plus grande méfiance car cette approche philologique a souvent conduit aux pires excès. Songeons, par exemple, à tous les cas où l'on a pu soutenir que la source d'un texte complet est un autre auteur, antérieur ou contemporain, dont on n'a presque rien conservé. Or dans le cas présent, il me semble que cette étude ne commet pas le genre d'excès que je déplore, non seulement parce que nous sommes en présence de deux textes complets dont l'un renvoie expressément à l'autre ${ }^{78}$, mais parce que nous savons également que Cicéron a lu et même traduit Xénophon. Au vu des nombreux recoupements entre les deux textes, et, dans certains cas, de la séquence même de ces recoupements, il me paraît raisonnable d'affirmer que les Mémorables comptent au nombre des sources du livre II du De natura deorum ${ }^{79}$. Si je suis parvenu à montrer que l'influence des chapitres théologiques des Mémorables sur le De natura deorum est beaucoup plus considérable que ne le donne à penser l'apparat

76. Voir Tableau, thème $\mathrm{n}^{\circ} 31$.

77. Voir Tableau, thème ${ }^{\circ} 30$. Contra, cf. Lois X 903c.

78. ND I 31 renvoie à Mem. IV 3, 13-14; ND II 18 renvoie à Mem. I 4, 8.

79. Dans la section de son commentaire consacrée aux sources du livre II du De natura deorum, Mayor (1883, p. xvi-xxiii) soutient que la principale source de Cicéron est Posidonius et il ne mentionne pas les Mémorables au nombre des sources. Mayor semble toutefois reconnaître que les Mémorables sont une importante source indirecte. Il affirme en effet, à propos du passage sur la divination (II $163 ;$; f. Tableau, thème $\mathrm{n}^{\circ} 31$ ), ce qui suit : « here too, as in so much of this treatise, the authority whom $C$. follows treads in the steps of Xen. Mem. I $4 \$ 15, I V$ $3 \S 12 \gg(1883$, p. 288$)$. 
des testimonia de l'édition Bandini-Dorion, il resterait à mettre en lumière, dans le prolongement des études de Sedley, l'étendue de l'influence exercée par les Mémorables sur l'ensemble des auteurs stoïciens qui ont conçu une théo-téléologie ${ }^{80}$.

J'aimerais, en terminant, souligner quelques points de divergence entre Xénophon et Cicéron. Nous avons vu, un peu plus tôt, qu'Épictète qualifie la nature de $\varphi \imath \lambda o ́ \zeta \omega o c$, alors que Xénophon réserve cette qualité à la divinité. De la même façon, Balbus, le porte-parole de la position stoïcienne, n'hésite pas à appliquer la métaphore artificialiste à la nature elle-même, en affirmant, par exemple, que la nature est un « ouvrier » (opifex, II 142), un « artisan » (artifex, II 58), qu'elle est « artiste » (artificiosa, II 57-58), « industrieuse » (sollertis, II 128, II 142) et que ses « ouvrages » (fabrica, II 138 ; opera, II 149) sont incroyables. Il parle également de « la providence de la nature » (providentiam naturae, II 140), comme si la providence relevait directement de la nature, et il soutient, de la même façon, que c'est la nature qui gouverne le monde (II 82, II 85, II 86). Dans la mesure où les stoïciens assimilent la nature à la divinité, il n'est pas étonnant qu'un porte-parole du stoïcisme parle de la providence de la nature et qu'il applique à la nature la métaphore artificialiste. Or Xénophon ne le fait jamais et il lui est impossible de le faire. Étant donné qu'il n'assimile pas la divinité à la nature, qu'il n'assimile pas non plus la théologie à la physique, et qu'il éprouve la plus grande méfiance pour ces « fous », ainsi qu'il les nomme ${ }^{81}$, qui se consacrent à l'étude de la nature, il ne pourrait pas, sans se contredire gravement, parler d'une providence de la nature ou encore appliquer la métaphore artificialiste à la nature. La providence est en effet le privilège exclusif des dieux et ce sont également les dieux qui sont comparés à des artisans doués d'intelligence ${ }^{82}$.

Dans la mesure où il règne un tel accord entre la théo-téléologie des Mémorables et celle des stoïciens, comment se fait-il que Socrate ne soutienne pas, comme les stoïciens, qu'il faut vivre en accord avec la nature ? Sans doute parce qu'il n'a pas conçu la possibilité d'assimiler la divinité à la nature. Il est à cet égard significatif que Socrate, dans les Mémorables, et que Balbus, chez Cicéron, n'entendent pas dans le même sens l'omniprésence qu' ils attribuent l'un et l'autre aux dieux ${ }^{83}$; pour Socrate, les dieux sont omniprésents pour autant qu'ils entendent et voient toutes choses, alors que pour Balbus et les stoïciens l'omniprésence des dieux est à entendre comme la coextensivité des

80. Meijer (2007, p. 19) affirme que Xénophon fut une source importante pour Zénon, mais non pour les stoïciens ultérieurs. Meijer n'avance cependant aucun argument pour justifier cette affirmation qui me paraît démentie par l'influence déterminante que Xénophon a exercée sur Arrien, le rédacteur des Entretiens et du Manuel d'Épictète.

81. Cf. Mem. I 1, 11 ; IV 7, 6.

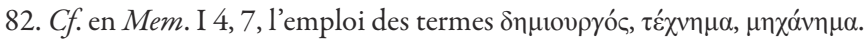

83. Cf. Tableau, thème no 33 . 
dieux à la nature. Cette différence entre Socrate et les stoïciens, en ce qui a trait aux rapports entre la divinité et la nature, se vérifie également dans le cas des « lois divines » de Socrate et des « lois naturelles » des stoïciens. Dans le chapitre qui suit immédiatement Mem. IV 3, Socrate affirme qu'il existe des lois non écrites qui ont pour caractéristiques d'être d'origine divine, d'être universelles, de valoir pour toutes les époques et d'entraîner un châtiment automatique pour celui qui les transgresse ${ }^{84}$. Ces quatre caractéristiques des lois non écrites sont également présentes dans la description que Cicéron donne de la loi naturelle :

Il existe certes une vraie loi, c'est la droite raison; elle est conforme à la nature (naturae congruens), répandue chez tous les hommes; elle est immuable et éternelle ; ses ordres appellent au devoir ; ses interdictions détournent de la faute. Si toutefois elle n'adresse jamais en vain aux honnêtes gens ses ordres et ses interdictions, elle ne peut, par ces moyens, faire impression sur les malhonnêtes. C'est un sacrilège que de la remplacer par une loi contraire ; il est interdit de n'en pas appliquer une seule disposition ; quant à l'abroger entièrement, personne n'en a la possibilité. Ni le sénat, ni le peuple ne peuvent nous soustraire à l'autorité de cette loi ; il est inutile de chercher un Sextus Aelius pour l'expliquer et l'interpréter; elle sera la même à Rome et à Athènes; la même maintenant et plus tard. Bref, cette loi unique, éternelle et immuable s'imposera à toutes les nations et à tous les temps, et un seul dieu commun à tous sera comme l'éducateur et le chef de tous. C'est lui qui a fait cette loi, qui l'interprète et nous l'a proposée. L'homme qui refusera de lui obéir devra se fuir lui-même et, comme il a méprisé la nature humaine, il subira les plus cruels châtiments, même au cas où il aurait échappé à tout ce que l'on considère comme un supplice ${ }^{85}$.

Socrate ne pourrait pas affirmer, comme le fait Cicéron, que cette loi d'origine divine est conforme à la nature ; l'aurait-il fait qu' il aurait par le fait même reconnu que la nature est prescriptive et que l'on peut donc déduire une éthique de la nature. Or Socrate s'est détourné de l'étude de la nature ${ }^{86}$ précisément parce qu'il ne croyait pas qu'elle pût fournir de réponse à la question de savoir comment il fallait vivre. Socrate n'aurait donc jamais pu souscrire au mot d'ordre du stoïcisme, « vivre conformément à la nature ».

84. Cf. Mem. IV 4, 19-25.

85. Resp. III 22, 33 (trad. Bréguet 1980). Taylor (1998, p. 80) évoque la possibilité que ce passage du De republica soit en fait, de la part de Cicéron, une imitation de Mem. IV 4.

86. Taylor (1998, p. 79-80) rappelle à juste titre que les stoïciens étaient embarrassés par l'indifférence de Socrate à l'endroit de la physique (cf. Mem. I 1, 16), dans la mesure où cela revient à dire que l'éthique ne peut pas être dérivée de la nature. La réponse des stoïciens consisterait à montrer que Socrate lui-même, en Mem. I 4 et IV 3, déduit des considérations morales de l'étude de la nature. 


\section{BIBLIOGRAPHIE}

Ademollo, F. 2012 : « The Platonic Origins of Stoic Theology », Oxford Studies in Ancient Philosophy, 43 (2012), p. 217-243.

Aubonnet, J. 1960 (éd. et trad.) : Aristote, Politique, Livres I-II, Paris, 1960 (Collection des Universités de France. Série grecque, 147).

Auvray-Assayas, C. 2002 (trad.) : Cicéron, La nature des dieux, Paris, 2002 (La roue à livres, 42).

Bandini, M. \& L.-A. Dorion 2000 (éd. et trad.) : Xénophon, Les Mémorables : tome I : Introduction générale, Livre I, Paris, 2000 (Collection des Universités de France. Série grecque, 399).

- 2011 (éd. et trad.) : Xénophon, Les Mémorables : tome II, 2 : Livre IV, Paris, 2011 (Collection des Universités de France. Série grecque, 478).

Barone, M. 1907 : «Senofonte e gli Stoici », Atene e Roma, 101 (1907), p. 145-151.

Borneclue, H. 1921 (trad.) : Cicéron, L'Orateur, Du meilleur genre d'orateurs, Paris, 1921.

BréGuet, E. 1980 (éd. et trad.) : Cicéron, La République, Livres II-VI, Paris, 1980 (Collection des Universités de France. Série latine, 6).

Charlton, W. 1991 : « Xenophon's Socrates as a philosopher », dans K. Boudouris (éd.), The Philosophy of Socrates, vol. 1, Athens, 1991 (Meletes stin helliniki filosofia, 5), p. 86-90.

Decleva Caizzi, F. 1966 (éd.) : Antistene, Fragmenta, Milano, 1966 (Testi e Documenti per lo studio dell'antichità, 13).

DeFilippo, J. \& Mitsis P. T. 1994 : « Socrates and Stoic Natural Law », dans P. Vander Waerdt (éd.), The Socratic Movement, Ithaca \& London, 1994, p. 252-271.

Diès, A. 1927 : Autour de Platon : essais de critique et d'histoire : vol. 1, Les voisinages, Socrate : vol. 2, Les dialogues, Esquisses doctrinales, Paris, 1927 (Bibliothèque des archives de philosophie).

- 1956 (éd. et trad.) : Platon, Les Lois, Livres VII-X, Paris, 1956 (Collection des Universités de France. Série grecque, 127).

DüMmLeR, F. 1889 : Akademica. Beiträge zur Literaturgeschichte der sokratischen Schulen, Giessen, 1889.

Erler, M. 2002 : «Stoic oikeiosis and Xenophon's Socrates », dans T. Scaltsas \& A.S. Mason (éd.), The Philosophy of Zeno : Zeno of Citium and his Legacy, Larnaka, 2002, p. 239-257.

Festugière, A.-J. 1949 : La révélation d'Hermès Trismégiste : vol. 2, Le Dieu cosmique, Paris, 1949 (Études bibliques).

Gilbert, W. 1891 (éd.) : Xenophontos Apomnemoneumata = Xenophontis Commentarii, Leipzig, 1891 (Bibliotheca scriptorum graecorum et romanorum Teubneriana).

Goulet, R. 1999 (trad.) : Diogène Laërce, Livre VII : « Zénon », dans M.-O. Goulet Cazé (éd.), Vies et doctrines des philosophes illustres, Paris, 1999 (La Pochotèque), p. 773-917. 
JAEger, W. 1943 : Paideia : The Ideals of Greek culture : vol. 2, In search of the divine centre, transl. by G. Highet from the German Manuscript, New York, 1943.

- 1966 : À la naissance de la théologie : Essai sur les Présocratiques, Paris, 1966 (Cogitatio fidei, 19).

JoËL, K. 1893 : Der echte und der xenophontische Sokrates, Berlin, 1893.

Krohn, A. 1875 : Sokrates und Xenophon, Halle, 1875.

LAKs, A. 1983 (éd.) : Diogène d'Apollonie, La dernière cosmologie présocratique, édition, traduction et commentaire des fragments et des témoignages, Lille, 1983 (Cahiers de Philologie, 9).

LinCKe, K. 1906 : « Xenophon und die Stoa », Neue Jahrbücher für das klassische Altertum, Geschichte und deutsche Literatur, 17 (1906), p. 673-691.

Long, A. A. 1988 : « Socrates in Hellenistic Philosophy », Classical Quarterly, 38/1 (1988), p. 150-171.

Long, A. G. 2013 (éd.) : Plato and the Stoics, Cambridge, 2013.

Mayor, J. B. 1883 (éd.) : M. Tullii Ciceronis De natura deorum, together with a new collation of several of the english mss. by J. H. Swainson, vol. 2, Cambridge, 1883.

McPherran, M. 1994 : « Socrates on Teleological and Moral Theology », Ancient Philosophy, 14/2 (1994), p. 245-262.

Meijer, P. A. 2007 : Stoic Theology : Proofs for the Existence of the Cosmic God and of the Traditional Gods, including a commentary on Cleanthes « Hymn on Zeus », Delft, 2007.

Mondésert, C. 1965 (trad.) : Clément d'Alexandrie, Le Pédagogue, vol. 2, Paris, 1965 (Sources chrétiennes, 108).

Powers, N. 2009 : « The Natural Theology of Xenophon's Socrates », Ancient Philosophy, 29/2 (2009), p. 249-266.

Reydams-Schils, G. 2013 : « The Academy, the Stoics and Cicero on Plato's Timaeus », dans A. G. Long (éd.), 2013, p. 29-58.

SCHLeIERmacher, F. D. E. 1840 : « The worth of Socrates as Philosopher », dans W. Smith (éd.), ПMATSN : The Apology of Socrates, the Crito and part of the Phaedo, with notes from Stallbaum, Schleiermacher's introductions, A Life of Socrates and Schleiermacher's essay on The worth of Socrates as a philosopher, Londres, 1840, p. CXXIX-CLV.

SEDleY, D. N. 2005 : « Les origines des preuves stoïciennes de l'existence de Dieu », Revue de métaphysique et de morale, 48/4 (2005), p. 461-487.

- 2007 : Creationism and its Critics in Antiquity, Berkeley/London, 2007 (Sather Classical Lectures, 66).

- 2008 : « Socrates' Place in the History of Teleology », Elenchos : rivista di studi sulpensiero antico, 29/2 (2008), p. 317-334.

TAYlor, C. C. W. 1998 : Socrates, Oxford, 1998 (Past Masters).

TheILER, W. 1925 : Zur Geschichte der teleologischen Naturbetrachtung bis auf Aristoteles, Zürich, 1925.

Viano, C. 2001 : « La cosmologie de Socrate dans les Mémorables de Xénophon », dans G. Romeyer Dherbey \& J.-B. Gourinat (éd.), Socrate et les socratiques, Paris, 2001 (Bibliothèque d'histoire de la philosophie), p. 97-119.

Vlastos, G. 1991 : Socrates : Ironist and Moral Philosopher, Ithaca, 1991 (Cornell Studies in Classical Philology, 50). 